\title{
Scale dependency of fractional flow dimension in a fractured formation
}

\author{
Y.-C. Chang ${ }^{1}$, H.-D. Yeh ${ }^{1}$, K.-F. Liang ${ }^{2}$, and M.-C. T. Kuo ${ }^{2}$ \\ ${ }^{1}$ Institute of Environmental Engineering, National Chiao Tung University, Hsinchu, Taiwan \\ ${ }^{2}$ Department of Mineral and Petroleum Engineering, National Cheng Kung University, Tainan, Taiwan
}

Received: 25 January 2011 - Published in Hydrol. Earth Syst. Sci. Discuss.: 22 February 2011

Revised: 15 June 2011 - Accepted: 30 June 2011 - Published: 13 July 2011

\begin{abstract}
The flow dimensions of fractured media were usually predefined before the determination of the hydraulic parameters from the analysis of field data in the past. However, it would be improper to make assumption about the flow geometry of fractured media before site characterization because the hydraulic structures and flow paths are complex in the fractured media. An appropriate way to investigate the hydrodynamic behavior of a fracture system is to determine the flow dimension and aquifer parameters simultaneously. The objective of this study is to analyze a set of field data obtained from four observation wells during an 11-day hydraulic test at Chingshui geothermal field (CGF) in Taiwan in determining the hydrogeologic properties of the fractured formation. Based on the generalized radial flow (GRF) model and the optimization scheme, simulated annealing, an approach is therefore developed for the data analyses. The GRF model allows the flow dimension to be integer or fractional. We found that the fractional flow dimension of CGF increases near linearly with the distance between the pumping well and observation well, i.e. the flow dimension of CGF exhibits scale-dependent phenomenon. This study provides insights into interpretation of fracture flow at CGF and gives a reference for characterizing the hydrogeologic properties of fractured media.
\end{abstract}

\section{Introduction}

For the determination of the hydrogeologic parameters, the traditional methods usually assume that the flow dimensions are predefined along with assumptions of homogeneity and isotropy before analyzing hydraulic test data. However, it will be normally the circumstance that no presumption about

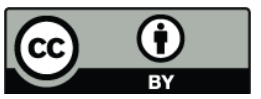

Correspondence to: H. D. Yeh

(hdyeh@mail.nctu.edu.tw) the dimension of the flow system can be made with confidence (Chakrabarty, 1994). In addition, the fractional flow dimension of the fracture zones is related to the connectivity of the fracture system, spatial and temporal variations of flow dimension; therefore, it may provide information on possible interconnections of major fracture zones (Acuna and Yortsos, 1995; Leveine et al., 1998; Leveinen, 2000). Since the hydrological, geothermal, and petroleum resources are plentiful in fractured media, it is important to determine the hydraulic parameters and the flow dimension simultaneously.

When analyzing data from the hydraulic test, it is difficult to choose an appropriate flow dimension in a fractured formation system. The flow geometry may be considered as a three-dimensional (3-D) spherical flow if the fracture density is large and its distribution is isotropic. On the other hand, a one-dimensional (1-D) or two-dimensional (2-D) flow model would probably be preferred (Barker, 1988) if the fracture density is low and its distribution is anisotropic. Theis (1935) presented an analytical solution to describe the radial flow with a line source, while it would be more appropriate to assume the cylindrical flow model is 2-D. The Theis model has been found to be inconsistent with some drawdown curves from fractured medium (Hamm and Bidaux, 1996; Leveinen, 2000; Le Borgne et al., 2004) and linear flow has been recognized in some fractured formations (Jenkins and Prentice, 1982). For fractured rocks, however, the flow dimensions may vary from 1-D to fully 3-D situations and they also include intermediate non-integer dimensions (Barker, 1988). Some models were proposed to describe the behavior of fracture systems (e.g. Barker, 1988; Chang and Yortsos, 1990; Acuna and Yortsos, 1995; Lods and Gouze, 2008). Barker (1988) developed a generalized radial flow (GRF) model for hydraulic tests in fractured formations by regarding the dimension of the flow as a parameter. Both integer and non-integer dimensions are therefore possible in the GRF model. Walker and Roberts (2003) indicated that the flow dimension is not necessarily a simple

Published by Copernicus Publications on behalf of the European Geosciences Union. 
function of radial distance. They mentioned that flow geometry and heterogeneity are interchangeable when interpreting the flow dimension based on the assumption that hydrogeologic properties are function of radial distance. Chen and Liu (2007) pointed out that the determination of apparent flow dimensions should consider all other knowledge of the system in order to construct a meaningful conceptual model of the system when commenting on the article by Walker and Roberts (2003).

For the flow dimension of fractured formation, KuuselaLahtinen et al. (2003) used the GRF model to examine the possibility in characterizing the hydrogeologic properties of fractured formation by the flow dimension determined from constant pressure injection tests. They demonstrated that there is a systematic trend in their results with higher dimensions corresponding to somewhat higher conductivities and clearly higher values of specific storage. Several cases in their study yielded a consistently acceptable fit in a varied range of flow dimension. Their explanation is that the injection flow is not sufficiently instantaneous at the beginning; therefore, this part of the injection flow curve can not be used in the curve fitting. The problem of such non-unique fits may be caused by the use of flow dimension being equal to 2, 2.5 and 3, rather than any arbitrary (non-integer) value in the type-curve fitting. In addition, the vertical flows might be produced near their tested boreholes which had $10 \mathrm{~m}$ and $2 \mathrm{~m}$ packer spacing in the depth ranging from 300 to $450 \mathrm{~m}$. The GRF model does not consider the vertical flow and thus it may not be appropriate to apply it in analyzing their sample data. The validity of their conclusion is therefore dubious because there is a trend in the results with higher dimensions corresponding to higher conductivities and specific storage. Le Borgne et al. (2004) described the average scaling properties of the spatial and temporal evolution of the drawdown cone in response to pumping in a heterogeneous fractured aquifer. They verified the fractional flow models presented by Barker (1988) and Acuna and Yortsos (1995) and obtained consistent fractional flow dimension from each of 7 observation wells. Walker et al. (2006) applied a numerical Monte Carlo analysis of an aquifer test for three stochastic models (multivariate Gaussian, fractional Brownian motion and percolation network) to simulate heterogeneous fields of transmissivity. They further examined the behavior of the flow with non-integer dimensions and their results indicated that the flow dimension may be useful in selecting hydrogeologic parameters in heterogeneous aquifers. Based on the previous work of Barker (1988) and Butler and Zhan (2004), Audouin and Bodin (2008) proposed new semi-analytical solutions for interpreting the cross-borehole slug tests with considering the fractional flow dimension of the aquifer and inertial effects at both the test and observation wells. Rafini and Larocque (2009) explored the use of flow dimensions in interpreting the fractional flow behaviors. They indicated that Barker's theory can be successfully applied to a discontinuum. Verbovšek (2009) addressed the difference between flow dimension and fractal dimension. The former, defined as a parameter in the GRF model, reflects the deficit or excess of interconnected flow paths in fractured rocks compared to one-, two-, or three-dimensionally connected networks (Leveinen, 2000). The latter characterizes a property of fracture networks obtained from the fracture traces in outcrops. He further analyzed the flow dimensions of different dolomite aquifers in Slovenia. The analyses of flow dimension of 72 pumping tests were performed using AQTESOLV based on the GRF model. The results show that there is no correlation between flow dimensions and fractal dimensions of dolomites and the flow dimensions are lower than the corresponding fractal dimensions in Slovenia. Rehbinder (2010) further extended Barker's analysis to develop the analytical solutions for Dirichlet's and Neumann's conditions at the boundary of a finite well. He demonstrated that the boundary value problems originating from the generalized radial flow model can be solved in closed forms for arbitrary boundary conditions and for a well of finite extent.

In the past, hydrogeologists often determined the flow dimension and hydrogeologic properties of the fractured aquifers using graphical methods from the analysis of the observed drawdown data. Based on a straight-line plot technique, Chakrabarty (1994) presented a fractional dimension analysis of constant rate interference tests in fractured rocks. Leveinen et al. (1998) utilized the GRF type curves to characterize the hydrogeologic properties of an aquifer in Finland comprising two subvertical facture zones. Leveinen (2000) formulated a composite analytical model with a source term that involves concrete parameters when the flow dimension is of fractional values. He applied the resulting analytical solution to analyze pumping test data in a fractured medium in south central Finland using type curve method. However, a good match to the Barker's solution by the graphical approach was practically impossible because there could be infinite type curves for the case of non-integer flow dimensions. In addition, graphical approaches may introduce extra errors during the curve fitting procedures.

In addition to the graphical methods, the hydrogeologic parameters can also be determined from some numerical methods. Yeh (1987) utilized the nonlinear least-squares and finite-difference Newton's method to determine the aquifer parameters and gave a fairly intensive literature review on the determination of the aquifer parameters (e.g. Rai, 1985; Czarnecki and Craig, 1985; Mukhopadhyay, 1985; Sen, 1986). However, two problems may arise when using such a gradient-type method to solve the NLS equations. First, nonconvergence is a common problem in NLS if the guessed parameter values are not close to the target values. Second, these methods may yield poor results if inappropriate increment is used when applying the finite difference formula to approximate the derivative terms.

In recent years, the global optimization methods based on heuristic search techniques have emerged rapidly. Simulated annealing (SA) is one of the major representatives of these 


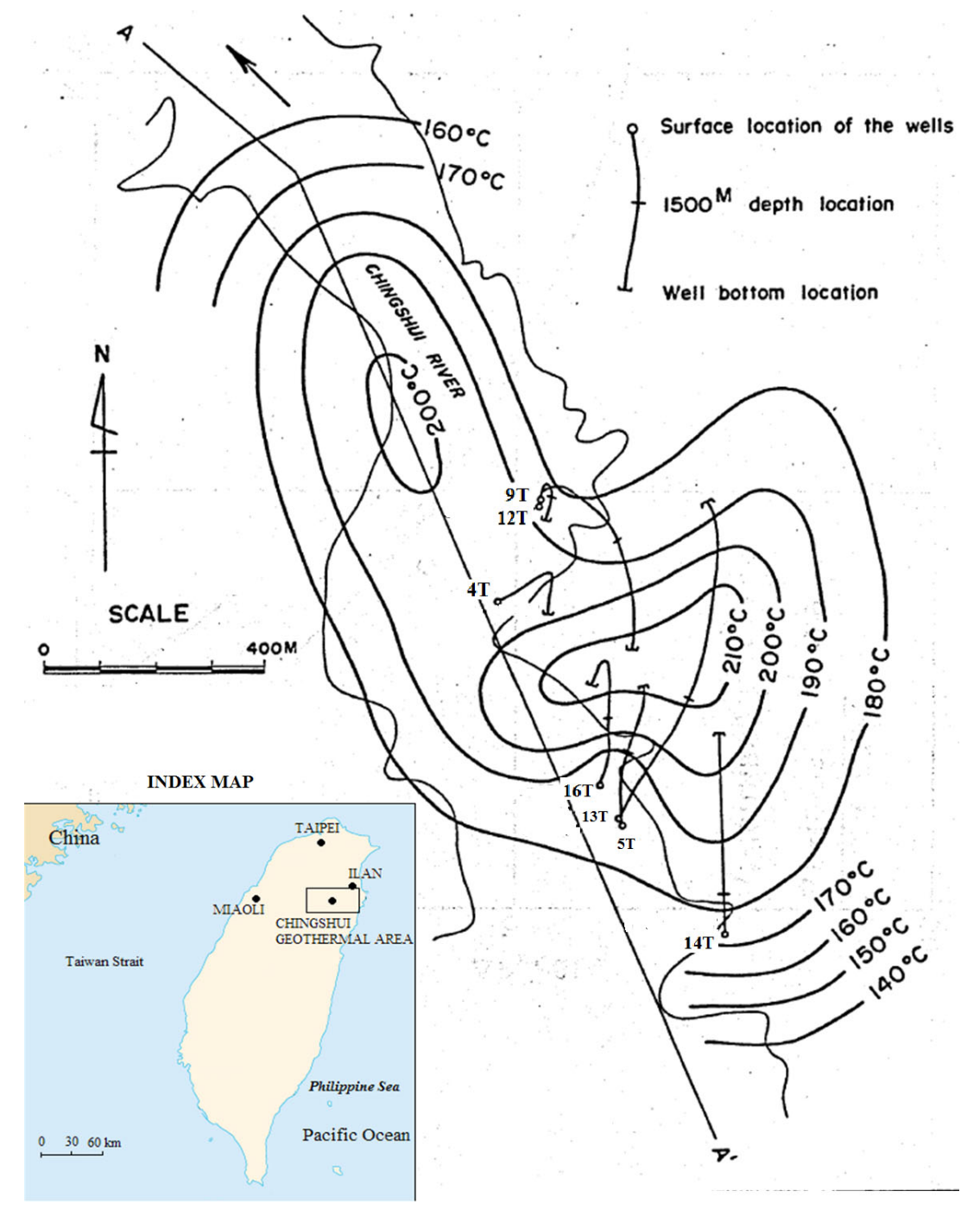

Fig. 1. Location of the CGF in Taiwan (adapted from Chang and Ramey, 1979).

optimization methods. The theory of SA was developed by Metropolis et al. (1953). They introduced a simple algorithm to incorporate the idea of the behavior of a particle system in thermal equilibrium into numerical calculations of equation state. SA was applied to solve the optimization problems in many fields; it is also useful in the determination of the hydrogeologic parameters. Huang and Yeh (2007) used SA and sensitivity analysis to determine the best-fit aquifer parameters of the leaky and unconfined aquifer systems. Yeh et al. (2007) employed SA and genetic algorithm to determine aquifer parameters of leaky aquifer systems. The major advantages of SA is its property of using descent strategy but allowing random ascent moves to avoid possible trap in a local optimum.

The Chingshui geothermal field (CGF) is a productive geothermal in Taiwan. It is worth determining its hydraulic parameters for assessing its hydrological or geothermal re- sources. The objective of this study is to characterize the CGF using GRF model, where there is no restriction on the flow dimension of CGF, verified as an adequate model for describing the hydraulic behavior in fractured media (see, e.g. Le Borgne et al., 2004; Rafini and Larocque, 2009; Verbovšek, 2009). In addition, SA is employed as an optimization algorithm and embedded in the GRF model to determine the hydrogeologic parameters of CGF which is a well-developed fractured formation. We found that the flow tends to be planar (one-dimensional) near the pumping source, cylindrical (two-dimensional) within the intermediate distance, and spherical (three-dimensional) at certain distance from the source. This suggests that the fractional flow dimension of CGF is scale-dependent. 


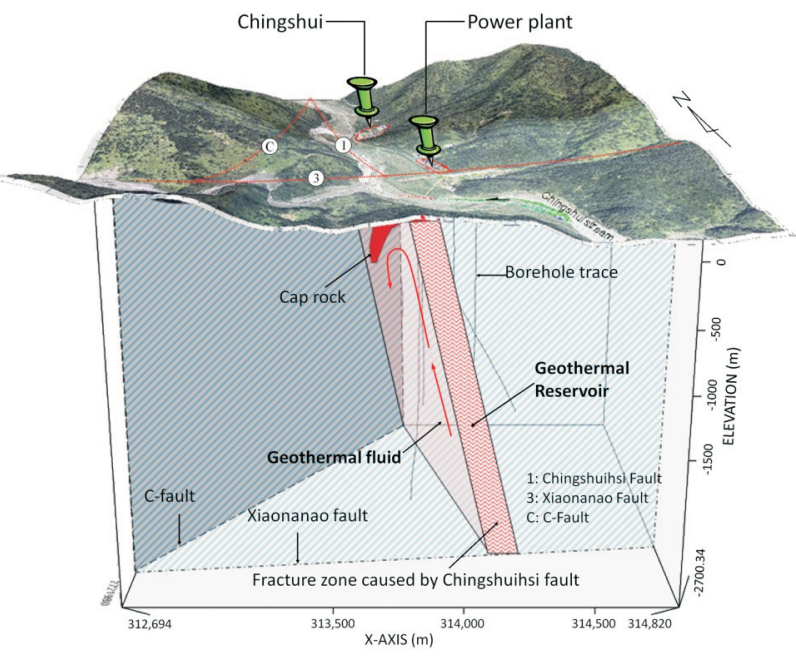

Fig. 2. The cross-sectional map of the inferred hydrologic featuresof the Chingshui hydrothermal system (Tong et al., 2008)

\section{Site description and data collection}

About a hundred hot springs, classified as volcanic or nonvolcanic hot springs, are found in Taiwan. The non-volcanic hot springs are usually located in both the sedimentary province and the metamorphic terrains of Taiwan. The CGF is in the metamorphic terrain and situated at the northeast portion of Taiwan as shown in Fig. 1. This field was first selected by a mining research organization for reconnaissance survey of geothermal resources in 1973. Further exploration was undertaken by a petroleum company in 1976 to explore a usable geothermal resource with greater production for power generation. Production in the liquid-dominated CGF is largely from a fractured formation.

The CGF is composed of dark-gray and black slates, namely the Miocene Lushan Formation which can be divided lithologically into the Jentse, Chingshuihu, and Kulu Members. The Jentse Member is constructed mainly by metasandstones intercalated in slates, while the underlying Chingshuihu and Kulu Members consist mostly of slates (Tseng, 1978; Chiang et al., 1979).

The cross-sectional map of the Chingshui hydrothermal system is presented in Fig. 2. There is a normal, NW-SE striking Chingshuihsi fault along the Chingshui River in the CGF site. The most convex of the NW-SE thrust faults is found around this geothermal field. It is postulated that the shear folding tectonic movements might have occurred with a greater tensile stress around the Chingshui geothermal area and created well-developed fractures in the slates. In addition, The CGF is situated at a monocline structure, which is cut internally by numerous thrust faults that essentially trend parallel to the bedding (NW-SE) and are lightly curved; the most important ones are the Tashi, Hsiaonanao and Hanhsi faults, shown in Fig. 3 (Su, 1978; Hsiao and Chiang, 1979).
There is clear evidence to consider that the geothermal reservoir is fracture dominated. Faults, joints, and other extensive fractures provide the conduits for the geothermal fluid flow due to the poor porosity and permeability of the slates. Predominant joints, which are almost aligned perpendicular to the strike of the strata, are found densely developed in the sandy Jentse Member. Figure 4 shows the rose diagram and contour diagram for 67 joints measured at an outcrop of the Jentse member nearby the CGF (Tseng, 1978). The most prominent set of joints strikes northwest and dips between $75^{\circ}$ and $90^{\circ}$ to the southwest. A less conspicuous set strikes northeast and dips steeply northwest. The trend of the Chingshui River is almost parallel to that of the joints. Its bed is cut through the slates, which present well-developed fractures. In the geothermal field, there are numerous hot springs and fumaroles along the river. It is reasonable to interpret that the riverbed is the area where the major open fractures reach the surface.

Subsurface data indicate that geothermal production at Chingshui is largely from a fracture zone in the steeply dipping Jentse Member (Hsiao and Chiang, 1979). Structural analyses indicate that this member presents predominant, well-developed, steeply dipping joints striking between $\mathrm{N} 25^{\circ} \mathrm{W}$ and $\mathrm{N} 40^{\circ} \mathrm{W}$. According to Tseng (1978), outcrops near the area of thermal manifestations also reveal that faults run parallel for almost 100 to 150 meters striking between $\mathrm{N} 30^{\circ} \mathrm{W}$ and $\mathrm{N} 35^{\circ} \mathrm{W}$. However, Tseng (1978) did not provide the dip direction and the azimuth of the fault. From the analysis of geologic, gravity, and magnetotelluric data by Tong et al. (2008), the fault system is $\mathrm{N} 21^{\circ} \mathrm{W}$ and dips to $80^{\circ}$ to NE.

Both pressure buildup and aquifer test of wells in the CGF site were performed during 1979. Two preliminary aquifer tests were conducted to determine whether detectable pressure responses would be available. The third aquifer test presented a comprehensive set of information for the CGF site and was conducted to determine the transmissivity and storage coefficient of Chingshui geothermal reservoir for the initial assessment of geothermal resources in deliverability and reserves (Chang and Ramey, 1979). During the aquifer test, the well 16T was produced and pressure responses were observed in other four wells. Hot water production rate ranged from 80000 to $83500 \mathrm{~kg} \mathrm{~h}^{-1}$ was measured in a weir during the 11-day interference test. The total fluid production rate was calculated from the hot water production rate using energy-balance criteria for flashing water. During the test, the wellhead pressure, water production rate and total fluid production rate at the flowing well $16 \mathrm{~T}$ were stabilized at 3.59 bars, $80000 \mathrm{~kg} \mathrm{~h}^{-1}$ and $105000 \mathrm{~kg} \mathrm{~h}^{-1}$, respectively. The aquifer thickness $B$ is about $300 \mathrm{~m}$. Wellhead pressures were monitored at all the observation wells except $5 \mathrm{~T}$ and $13 \mathrm{~T}$. These two wells appeared to be unreliable due to the equipment malfunction. The distances between the pumping well $16 \mathrm{~T}$ and the observation wells $4 \mathrm{~T}, 9 \mathrm{~T}, 12 \mathrm{~T}$ and $14 \mathrm{~T}$ are 175, 300, 90 and $330 \mathrm{~m}$, respectively (Fan et al., 


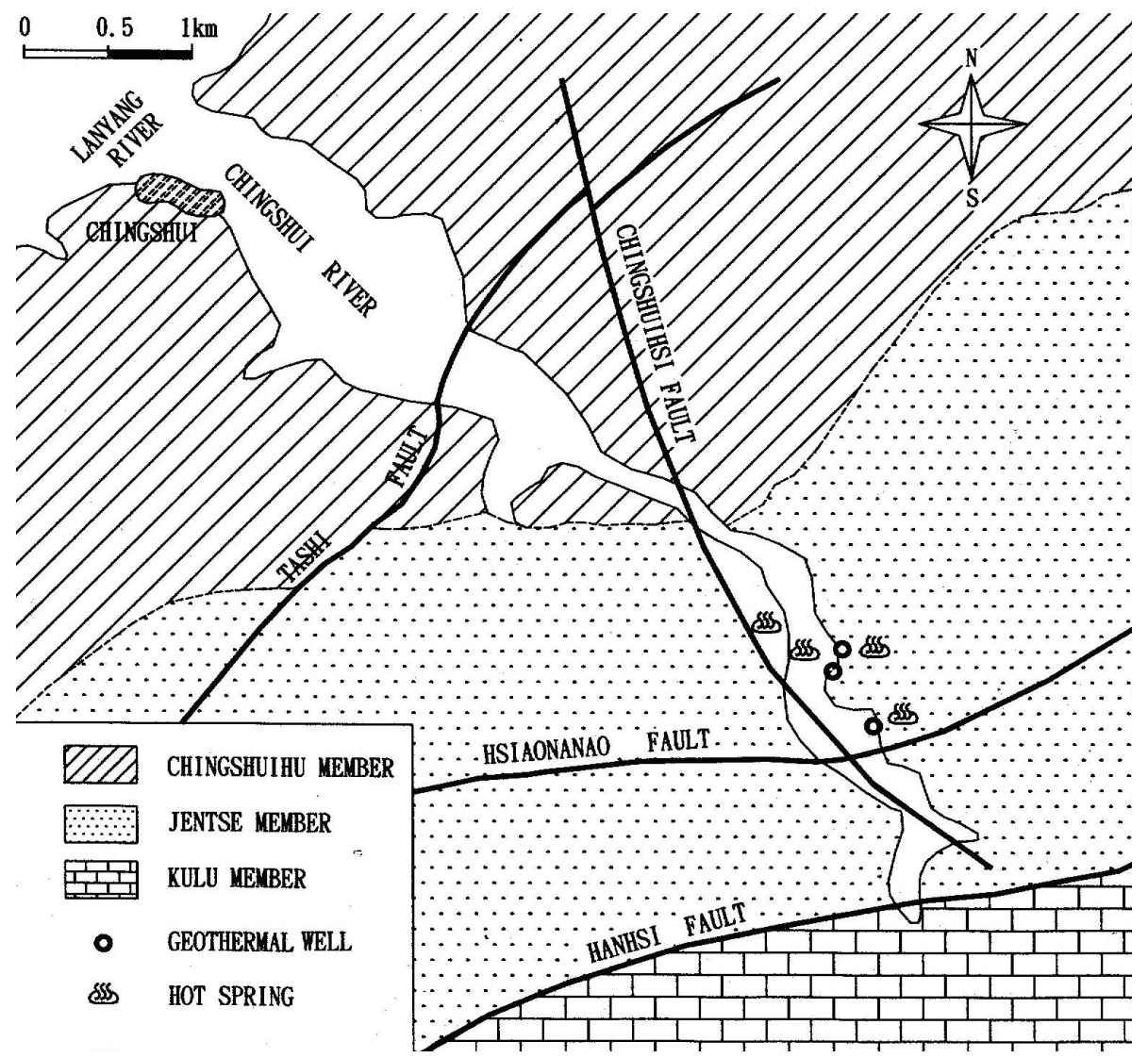

Fig. 3. Geological map of the Chingshui geothermal area describing Chingshuihu, Jentse, and Kulu members of the Miocene Lushan Formation (Su, 1978; Hsiao and Chiang, 1979)

2005). Equivalent to $80 \mathrm{th}^{-1}$ of hot-water in well $16 \mathrm{~T}$ was measured and the total production rate of well stream was $1.89 \mathrm{~m}^{3} \mathrm{~min}^{-1}$ during the test. The wellhead pressure for the observation wells is measured at about 24 hourly intervals. The set of observed data is presented in Table 1 (Chang and Ramey, 1979). The differences in the wellhead pressure $\Delta p$ $\left(\mathrm{kg} \mathrm{cm}^{-2}\right)$ are converted into drawdown in meter.

The wells were drilled by the petroleum company from 1976. The system reached the thermal equilibrium between the borehole fluid and the formation before the tests were undertaken in 1979. Accordingly, there was no variation in the borehole fluid temperature during the aquifer test. In addition, the temperature might not have minor influence on the results of the tests (Pickens et al., 1987).

\section{Methodology}

\subsection{Generalized radial flow model}

Barker (1988) developed a solution for $n$-dimensional radial flow in an infinite domain from an $n$-dimensional sphere source. The flow dimension of the radial flow may be integer or non-integer. Using Theis assumptions, Barker (1988) gave a generalized flow equation expressed in term of drawdown as:

$S_{s} \frac{\partial s}{\partial t}=\frac{K}{r^{n-1}} \frac{\partial}{\partial r}\left(r^{n-1} \frac{\partial s}{\partial r}\right)$

where $S_{s}$ is the specific storage of the fracture system; $K$ is the hydraulic conductivity; $n$ is the dimension of the fracture flow system; $r$ is the radial distance from the centre of the source; $t$ is the well production time. For the constant-rate condition, the solution can be written as:

$s(r, t)=\frac{Q r^{2 v}}{4 \pi^{1-v} \mathrm{~Tb}^{2-n}} \Gamma(-v, u)$

where

$v=1-\frac{n}{2}$

and

$u=\frac{S r^{2}}{4 T t}$

where $1 / u$ is the dimensionless time; $Q$ is the constant well production rate; $b$ is the extent of the flow region; $\Gamma(-v, u)$ is a complementary incomplete gamma function; $T$ is the 
Table 1. Aquifer Test in CGF (Chang and Ramey 1979).

\begin{tabular}{|c|c|c|c|c|c|c|c|c|c|c|c|c|c|c|c|}
\hline \multirow{4}{*}{ Time (h) } & \multicolumn{12}{|c|}{ Observation Wells } & \multirow{2}{*}{\multicolumn{3}{|c|}{$\begin{array}{c}\text { Flowing Well } \\
16 \mathrm{~T} \\
\end{array}$}} \\
\hline & \multicolumn{3}{|c|}{$4 \mathrm{~T}$} & \multicolumn{3}{|c|}{$9 \mathrm{~T}$} & \multicolumn{3}{|c|}{$12 \mathrm{~T}$} & \multicolumn{3}{|c|}{$14 \mathrm{~T}$} & & & \\
\hline & WHP* & $\Delta p^{* *}$ & $s^{* * *}$ & WHP & $\Delta p$ & $s$ & WHP & $\Delta p$ & $s$ & WHP & $\Delta p$ & $s$ & WHP & $\Delta p$ & \multirow{2}{*}{$\begin{array}{l}\text { Weir Water } \\
\text { Rate }\left(\mathrm{th}^{-1}\right)\end{array}$} \\
\hline & $\left(\mathrm{kg} \mathrm{cm}^{-2}\right)$ & (psi) & $\left(\mathrm{m}-\mathrm{H}_{2} \mathrm{O}\right)$ & $\left(\mathrm{kg} \mathrm{cm}^{-2}\right)$ & (psi) & $\left(\mathrm{m}-\mathrm{H}_{2} \mathrm{O}\right)$ & $\left(\mathrm{kg} \mathrm{cm}^{-2}\right)$ & (psi) & $\left(\mathrm{m}-\mathrm{H}_{2} \mathrm{O}\right)$ & $\left(\mathrm{kg} \mathrm{cm}^{-2}\right)$ & (psi) & $\left(\mathrm{m}-\mathrm{H}_{2} \mathrm{O}\right)$ & $\left(\mathrm{kg} \mathrm{cm}^{-2}\right)$ & (psi) & \\
\hline 0 & 12.09 & 172 & 0.00 & 9.70 & 138 & 0.00 & 13.15 & 187 & 0.00 & 9.35 & 133 & 0.00 & 18.14 & 258 & 0 \\
\hline 18.5 & 12.02 & 171 & 0.73 & 9.63 & 137 & 0.70 & 13.01 & 185 & 1.41 & 9.35 & 133 & 0.00 & 4.85 & 69 & 24 \\
\hline 42.5 & 11.81 & 168 & 2.93 & 9.49 & 135 & 2.11 & 12.80 & 182 & 3.52 & 9.14 & 130 & 2.11 & 4.08 & 58 & 83.5 \\
\hline 66.5 & 11.67 & 166 & 4.41 & 9.35 & 133 & 3.52 & 12.80 & 182 & 3.52 & 8.79 & 125 & 5.63 & 3.94 & 56 & 83.1 \\
\hline 90.5 & 11.67 & 166 & 4.41 & 9.14 & 130 & 5.63 & 12.66 & 180 & 4.92 & 8.79 & 125 & 5.63 & 3.94 & 56 & 83.1 \\
\hline 114.5 & 11.60 & 165 & 5.14 & 9.14 & 130 & 5.63 & 12.59 & 179 & 5.63 & 8.65 & 123 & 7.03 & 3.94 & 56 & 82 \\
\hline 138.5 & 11.53 & 164 & 5.87 & 9.14 & 130 & 5.63 & 12.52 & 178 & 6.33 & 8.51 & 121 & 8.44 & 3.94 & 56 & 82.4 \\
\hline 162.5 & 11.53 & 164 & 5.87 & 9.07 & 129 & 6.33 & 12.44 & 177 & 7.03 & 8.44 & 120 & 9.14 & 3.80 & 54 & 82.4 \\
\hline 186.5 & 11.46 & 163 & 6.61 & 9.00 & 128 & 7.03 & 12.37 & 176 & 7.74 & 8.37 & 119 & 9.85 & 3.80 & 54 & 81 \\
\hline 210.5 & 11.39 & 162 & 7.35 & 8.93 & 127 & 7.74 & 12.30 & 175 & 8.44 & 8.37 & 119 & 10.55 & 3.73 & 53 & 80 \\
\hline 234.5 & 11.39 & 162 & 7.35 & 8.93 & 127 & 7.74 & 12.30 & 175 & 8.44 & 8.23 & 117 & 11.25 & 3.66 & 52 & 80 \\
\hline 258.5 & 11.32 & 161 & 8.08 & 8.86 & 126 & 8.44 & 12.30 & 175 & 8.44 & 8.09 & 115 & 12.66 & 3.66 & 52 & 80 \\
\hline
\end{tabular}

* WHP: Wellhead pressure $* * \Delta p$ : Pressure difference $* * * s$ : Drawdown.

transmissivity; $S$ is the storage coefficient. When the flow dimension $n$ is equal to 2, Eq. (2) reduces to the equation introduced by Theis (1935) as:

$s(r, t)=\frac{Q}{4 \pi T} E_{1}(u) \quad$ for $n=2$

where $E_{1}(x)$ is the exponential integral.

Using the GRF model, the well is mathematically implemented as a plane for perfectly linear flow $(n=1)$ and $b$ equals the square root of the throughflow area at the source. The parameter $b$ is the thickness of the aquifer and the flow is cylindrical $(n=2)$. For spherical flow $(n=3)$, the term $b^{3-n}$ becomes unity, and the value of $b$ is therefore irrelevant.

\subsection{Simulated annealing}

The concept of SA is analogous to the physical annealing process which is to heat up an object from solid phase to liquid phase and then let it cool down slowly. As the temperature is reduced, the atomic energies decrease. As it is crystallized, the system energy of the object will be in the minimum state. Based on the annealing concept, SA was constructed for solving the optimization problems. During the calculation procedure, the system allows the solutions to escape from a local optimum. The temperature is increased to enhance the molecule mobility at the beginning of the process. Then the temperature is slowly decreased to form molecules as crystalline structures. The molecules have high activity when the temperature is high and the crystalline configurations have various forms. If the temperature is cooled properly, the crystalline configuration is in the most stable state; thus, the minimum energy level may be naturally reached. The concept and the process of SA are explained more detail in Kirkpatrick et al. (1983).

\subsection{Application of SA}

The hydrogeologic parameters of field data can be determined based on the analytical solution coupled with SA in minimizing the sum of square differences between the observed and predicted hydraulic heads. The first step in SA is to generate a trial solution for unknown parameters from a random number generator. Each parameter value has its own upper and lower bounds. Once the guessed parameter values are generated, Eq. (2) is used to calculate the hydraulic heads. At the beginning, the initial solution is considered as the current optimal solution. Then, SA generates new trial solutions and calculates its corresponding objective function value (OFV). The objective function is defined as

Minimize $\sum_{i=1}^{p}\left(\mathrm{Oh}_{i}-\mathrm{Eh}_{i}\right)^{2}$

where $\mathrm{Oh}_{i}$ and $\mathrm{Eh}_{i}$ are the observed and predicted heads, respectively, at different time and $p$ is the number of observed data.

With the OFV, the algorithm of SA checks the trial solution to see whether this one is a new optimum or not in the next step. If the OFV satisfies Metropolis criterion (Pham and Karaboga, 2000) described below, the current optimal solution is replaced by the trial solution. Otherwise, the algorithm will continue generating the new trial solution.

The Metropolis's criterion is given as (Metropolis et al., 1953):

$P_{\mathrm{SA}}\{\operatorname{accept} j\}=\left\{\begin{array}{l}1, \text { if } f(j) \leq f(i) \\ \exp \left(\frac{f(i)-f(j)}{T \mathrm{e}}\right), \text { if } f(j)>f(i)\end{array}\right.$

where $P_{\mathrm{SA}}$ is the accepted probability of the trial solution, $f(i)$ and $f(j)$ are the function value when $x=x_{i}$ and $x=x_{j}$, respectively, and $x_{i}$ and $x_{j}$ are the current best solution and neighborhood trial solution of $x$, respectively. Here $T \mathrm{e}$, a control parameter, is the current temperature.

The temperature value depends on the scale of the objection function $f$ of the problem. Kirkpatrick (1984) suggested that a suitable initial temperature $T_{0}$ is one that results in an average probability $\chi_{0}$ of a solution that increases the $f$ being accepted of about 0.8 . It can be determined by conducting an 


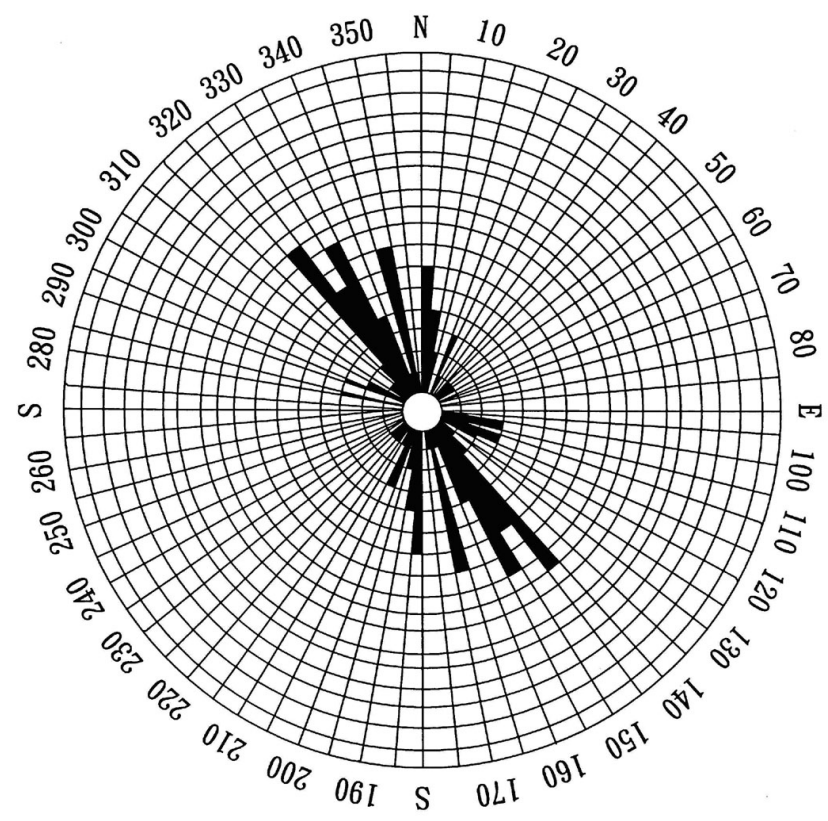

Note that $N$ represents the number of considered variables, $N \mathrm{~S}$ represents the number of steps at a specific temperature, and $N T$ represents the number of times through the loop. The new temperature is then

$T \mathrm{e}^{\prime}=R_{T \mathrm{e}} \times T \mathrm{e}$

The value of $R_{T \mathrm{e}}$ is constant and smaller than one (Pham and Karaboga, 2000). The temperature should be cooled properly to guarantee the resulting solution being the global optimal solution. The parameter estimation process will be terminated when the resulting solution satisfies the stopping criteria. Two criteria are considered in this study. The first one is to check whether the absolute difference between two OFVs obtained at two consecutive temperatures is less than $10^{-9}$ nine times successively. The second one is to check whether the total function evaluations exceed a chosen maximum evaluation, say $10^{6}$ in this study.

The standard error of estimate (SEE) is defined as

$\mathrm{SEE}=\sqrt{\frac{1}{v} \sum_{i=1}^{n} e_{i}^{2}}$

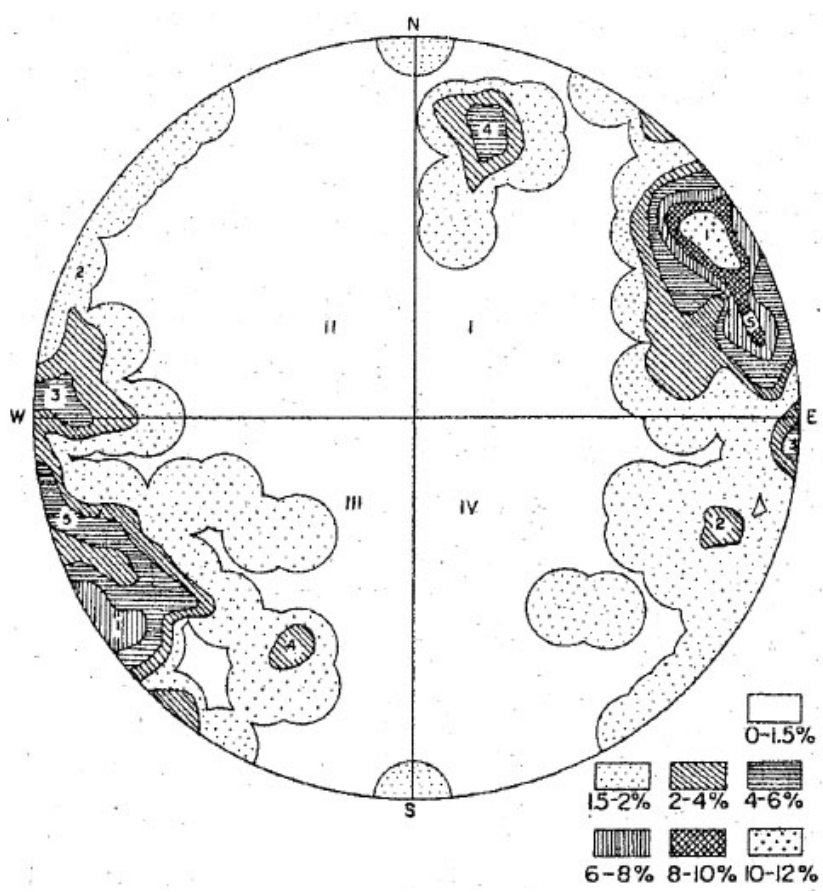

Fig. 4. (a) Rose diagram and (b) contour diagram of 67 joints of Jentse member in the Chingshui geothermal area (Tseng, 1978).

initial search in which all increases in $f$ are accepted and calculating the average objective increase in observed $\bar{\delta} f^{+}$. The initial temperature $T_{0}$ is then given by:

$T_{0}=-\left(\bar{\delta} f^{+}\right) / \ln \left(\chi_{0}\right)$

where $\bar{\delta} f^{+}$is the increase in $f$.

In $\mathrm{SA}$, after $N \times N T \times N \mathrm{~S}$ function simulations, the temperature $T \mathrm{e}$ is decreased by the temperature reduction factor $R_{T \mathrm{e}}$ even if no improvement in the optimum takes place. where $e_{i}$ is the difference between the observed drawdown and predicted drawdown and $v$ is the degree of freedom, which equals the number of observed data points minus the number of unknowns. (Note that here $v$ is 11-3 for GRF model).

\section{Data analyses and discussion}

The approach, based on the GRF model coupled with the SA algorithm, is used to analyze the test data from each observation well at the CGF site for simultaneously determining the flow dimension and hydrogeologic parameters of the CGF. A pumping test with 4 observation wells was conducted over a period of 10.8 days $(258.5 \mathrm{~h})$. Such a long pumping period produced the drawdowns ranged from $8.08 \mathrm{~m}$ to $12.66 \mathrm{~m}$ in the observation wells. The radii of influence ranging from $1600 \mathrm{~m}$ to $2400 \mathrm{~m}$ cover the entire CGF. Thus, those drawdown data should be able to interpret the field flow system and hydrogeologic properties of the CGF.

The geology of CGF can be regarded as homogenous because it has well-developed fractures in the slates. In addition, the formation of CGF is further considered as isotropic. An anisotropic analysis of the drawdown data from the observation wells is performed using the anisotropic model of Papadopulous (1965). The Papadopulous model is

$T_{x x} \frac{\partial^{2} s}{\partial x^{2}}+2 T_{x y} \frac{\partial^{2} s}{\partial x \partial y}+T_{y y} \frac{\partial^{2} s}{\partial y^{2}}+Q \delta(x) \delta(y)=S \frac{\partial s}{\partial t}$

where $T_{x x}, T_{x y}$, and $T_{y y}$ are the components of transmissivity tensor in the Cartesian coordinates and $s$ is the drawdown. Four sets of drawdown data obtained from the combination of three wells from the four observation wells and one set of 
drawdown data obtained from the composite wells as shown in Table 2 are analyzed using Papadopulous' model with known coordinates of the well locations. The coordinates of the observation wells measured from pumping well $16 \mathrm{~T}$ are $(-89 \mathrm{~m}, 150.65 \mathrm{~m})$ for $4 \mathrm{~T},(-79 \mathrm{~m}, 289 \mathrm{~m})$ for $9 \mathrm{~T},(55 \mathrm{~m}$, $71 \mathrm{~m})$ for $12 \mathrm{~T}$ and $(260 \mathrm{~m},-200 \mathrm{~m})$ for $14 \mathrm{~T}$. The angles of the wells can also be estimated from the coordinates of the well locations. The results of anisotropic analysis are also shown in Table 2 with $T_{\xi \xi}$ and $T_{\eta \eta}$ defined as the major and minor principal directional components of the transmissivity tensor, respectively and $\theta$ defined as the angle between the $\mathrm{x}$-axis and the direction of the major principal transmissivity. The parameters $T_{\xi \xi}, T_{\eta \eta}$ and $\theta$ are defined, respectively, as

$T_{\xi \xi}=\frac{1}{2}\left\{\left(T_{x x}+T_{y y}\right)+\left[\left(T_{x x}-T_{y y}\right)^{2}+4 T_{x y}^{2}\right]^{1 / 2}\right\}$

$T_{\eta \eta}=\frac{1}{2}\left\{\left(T_{x x}+T_{y y}\right)-\left[\left(T_{x x}-T_{y y}\right)^{2}+4 T_{x y}^{2}\right]^{1 / 2}\right\}$

and

$\theta=\arctan \left(\frac{T_{\xi \xi}-T_{x x}}{T_{x y}}\right)$

As mentioned above, the most prominent set of joints strikes are about $-50^{\circ}$ and $-65^{\circ}$ from the W-E direction (i.e. $\mathrm{N} 25^{\circ} \mathrm{W}$ and $\mathrm{N} 40^{\circ} \mathrm{W}$ ). The results demonstrate that the major transmissivities have similar directional components as the prominent joints in sets 1 to 3 . Theoretically, the major transmissivity and the prominent joints in all set of experiments are situated in the same direction. However, the directions in sets 4 and 5 are inconsistent with the direction of prominent set of joints in Fig. 4a. The major direction of transmissivity in set 4 is even perpendicular to the direction of prominent set of joints. The analysis of wells using anisotropic model implies that besides faults and joints, there might be a highly well-developed fracture or micro-fracture network in the field. The results demonstrate that the principal directions of transmissivities are different in all sets of wells and there is no obvious evidence to show the existence of anisotropy in this field. The GRF model is therefore applicable to the CGF because it is homogeneous and isotropic based on the field description and anisotropic analysis.

The estimated results for flow dimension and hydrogeologic parameters of CGF given in Table 3 are obtained from the proposed approach. The results obtained from Theis' model (i.e. $n=2$ case) are also provided in this table. The estimated results range from 1.31 to 2.27 for the flow dimension, $48.9 \times 10^{-3}$ to $99.9 \times 10^{-3} \mathrm{~m}^{2} \mathrm{~min}^{-1}$ for the transmissivity, and $3.64 \times 10^{-3}$ to $9.99 \times 10^{-3}$ for the storage coefficient. The average values of transmissivity and storage coefficient are $79 \times 10^{-3} \mathrm{~m}^{2} \mathrm{~min}^{-1}$ and $6.235 \times 10^{-3}$, respectively. The plots of the predicted drawdowns at different wells from Theis' model $(n=2)$ are compared with those from GRF model as shown in Fig. 5. Since the residual plot is an auxiliary tool to assess the goodness-of-fit of the model, the residuals calculated from Theis' and GRF models for different wells are further demonstrated in Fig. 6. The results show that there is no obvious difference in the residual plots obtained from Theis' and GRF models except the results of well $12 \mathrm{~T}$. As listed in Table 1, the aquifer test started at $18.5 \mathrm{~h}$ to $258.5 \mathrm{~h}$ and the drawdown data are observed during this period. Using the estimated aquifer parameters for each observation well in Table 3 and the definition of dimensionless time $(1 / u)$ in Eq. (4), the dimensionless time of these drawdown data falls in the ranges of 1.38 to $19.30,0.96$ to 13.46 and 0.59 to 8.27 for wells $4 \mathrm{~T}, 9 \mathrm{~T}$ and $14 \mathrm{~T}$, respectively. As shown in Fig. 7 (Barker, 1988), it is rather difficult to discriminate the curves of Theis' model and other models in this range because they all have very similar drawdown shapes. On the other hand, it is rather easy to distinguish the model for well $12 \mathrm{~T}$ from Theis model since its dimensionless time ranges from 5.5 to 76.6. In addition, as shown in Fig. 6c, the predicted drawdown at well $12 \mathrm{~T}$ from the GRF model has smaller residuals than those from the Theis' model. This indicates that the GRF model is more appropriate than the Theis' model for describing the CGF data. Note that large differences in parameter values would be obtained if the flow dimension is assumed to be 2 (i.e. Theis' model). Although the drawdown curves predicted form Theis' model and the GRF model have very similar shape in wells $9 \mathrm{~T}$ and $14 \mathrm{~T}$, the estimated values of transmissivity and storage are however significantly different for both models as listed in Table 3. The estimated aquifer parameters determined from GRF model are almost 2 and 5 times as large as the parameter values determined from Theis' model in well $9 \mathrm{~T}$ and $14 \mathrm{~T}$, respectively. Thus, those results indicate that it is inappropriate to pre-assume the flow dimension as 2 (e.g. using Theis' model) in the determination of hydrogeologic parameters.

\subsection{Weighted least squares}

A weighted objective function might be adopted in the regression analysis if the observed data have nonconstant variance (Berthouex and Brown, 2002) or the data have different relative reliability (Xu and Eckstein, 1995). The weights assigned in the weighted least squares are generally chosen to be inversely proportional to the values of independent or dependent variable (Berthouex and Brown, 2002, p. 331) or proportional to the quality of the data, i.e. higher weights reflect more reliable data points (Xu and Eckstein, 1995). The sensitivity analysis is performed to investigate the normalized sensitivities (Huang and Yeh, 2007) of the drawdown with respect to the flow dimension $n$ and the aquifer parameters $T$ and $S$. Figure 8 shows that the drawdown is sensitive to the changes of $n$ and $T$ except at the early period of the pumping. The normalized sensitivity of $S$ is relative small compared with those of $T$ and $n$. In addition, the normalized sensitivities of the drawdown with respect to $n, T$, and $S$ are continuously increased through the end of the pumping, indicating that the late-time drawdown data are more 
Table 2. Anisotropic analysis of the drawdown data from CGF.

\begin{tabular}{lccccc}
\hline \multirow{2}{*}{ Set } & 1 & 2 & 3 & 4 & 5 \\
\cline { 2 - 6 } & $9 \mathrm{~T}, 12 \mathrm{~T}, 14 \mathrm{~T}$ & $4 \mathrm{~T}, 12 \mathrm{~T}, 14 \mathrm{~T}$ & $4 \mathrm{~T}, 9 \mathrm{~T}, 12 \mathrm{~T}$ & $4 \mathrm{~T}, 9 \mathrm{~T}, 14 \mathrm{~T}$ & All wells \\
\hline$T_{\xi \xi}\left(\mathrm{m}^{2} \mathrm{~min}^{-1}\right)$ & 8.574 & 9.95 & 1.48 & 1.087 & 1.081 \\
$T_{\eta \eta}\left(\mathrm{m}^{2} \mathrm{~min}^{-1}\right)$ & $6.31 \times 10^{-4}$ & $5.0 \times 10^{-4}$ & $1.5 \times 10^{-3}$ & $1.4 \times 10^{-3}$ & $1.4 \times 10^{-3}$ \\
$\theta$ & $-36^{\circ}$ & $-443^{\circ}$ & $-86^{\circ}$ & $53^{\circ}$ & $-19^{\circ}$ \\
\hline
\end{tabular}

$T_{\xi \xi}$ and $T_{\eta \eta}$ are the major and minor principal directional components of the transmissivity tensor; $\theta$ is the angle between the $\mathrm{x}$-axis and the direction of the major principal transmissivity.

Table 3. The distances from pumping well and the estimated hydrogeologic parameters for $4 \mathrm{~T}, 9 \mathrm{~T}, 12 \mathrm{~T}$ and $14 \mathrm{~T}$ by SA.

\begin{tabular}{|c|c|c|c|c|c|c|}
\hline \multirow{2}{*}{$\begin{array}{l}\text { Observation } \\
\text { wells }\end{array}$} & \multicolumn{5}{|c|}{ Estimated hydrogeologic parameters } & \multirow{2}{*}{$\operatorname{SEE}\left(\mathrm{m}^{2}\right)$} \\
\hline & $r^{*}(\mathrm{~m})$ & model & $n$ & $T\left(\mathrm{~m}^{2} \min ^{-1}\right)$ & $S$ & \\
\hline \multirow{2}{*}{$12 \mathrm{~T}$} & \multirow{2}{*}{90} & GRF & 1.31 & $99.9 \times 10^{-3}$ & $9.99 \times 10^{-3}$ & 0.38 \\
\hline & & Theis & 2.0 & $40.2 \times 10^{-3}$ & $18.1 \times 10^{-3}$ & 0.39 \\
\hline \multirow{2}{*}{$4 \mathrm{~T}$} & \multirow{2}{*}{175} & GRF & 1.95 & $48.9 \times 10^{-3}$ & $5.13 \times 10^{-3}$ & 0.35 \\
\hline & & Theis & 2.0 & $46.2 \times 10^{-3}$ & $5.13 \times 10^{-3}$ & 0.33 \\
\hline \multirow{2}{*}{$9 \mathrm{~T}$} & \multirow{2}{*}{300} & GRF & 2.11 & $71.2 \times 10^{-3}$ & $3.64 \times 10^{-3}$ & 0.44 \\
\hline & & Theis & 2.0 & $37.8 \times 10^{-3}$ & $1.94 \times 10^{-3}$ & 0.41 \\
\hline \multirow{2}{*}{$14 \mathrm{~T}$} & \multirow{2}{*}{330} & GRF & 2.27 & $96.0 \times 10^{-3}$ & $6.54 \times 10^{-3}$ & 0.57 \\
\hline & & Theis & 2.0 & $20.6 \times 10^{-3}$ & $1.40 \times 10^{-3}$ & 0.54 \\
\hline
\end{tabular}

$r$ is the radial distance from pumping well to observation well.

critical than the early-time drawdown data. The weights $w_{i}=t_{i} / \sum_{i=1}^{11} t_{i}$, where $t_{i}$ denoted as $i-$ the production time, reflect the fact that the late-time data is more important to the observed drawdown than the early-time data. Table 4 lists the parameters estimated by the weighted least squares form data obtained at different observation wells. Apparently, the parameters estimated by the weighted least squares are not significantly different from those by the unweighted one as shown in Table 3. In addition, the new flow dimension also increases with the distance between the pumping well and the observation well.

\subsection{Robustness and reliability of SA}

For examining the robustness and reliability of SA in parameter identification, Yeh et al. (2007) and Huang et al. (2008) presented the sensitivity analyses of control parameters in SA for the parameter identification. They demonstrated that the use of different temperature reduction factors does not affect the results of the parameter identification. Table 5 shows the parameters and flow dimension of CGF determined form the data observed at $4 \mathrm{~T}, 9 \mathrm{~T}, 12 \mathrm{~T}$ and $14 \mathrm{~T}$ when the temperature
Table 4. The parameters estimated by weighted least squares.

\begin{tabular}{|c|c|c|c|c|c|}
\hline \multirow{2}{*}{$\begin{array}{l}\text { Observation } \\
\text { well }\end{array}$} & \multirow[t]{2}{*}{$r(\mathrm{~m})$} & \multicolumn{3}{|c|}{ Estimated hydrogeologic parameters } & \multirow{2}{*}{$\operatorname{SEE}\left(m^{2}\right)$} \\
\hline & & $n$ & $T\left(\mathrm{~m}^{2} \min ^{-1}\right)$ & $S$ & \\
\hline $12 \mathrm{~T}$ & 90 & 1.31 & $96.6 \times 10^{3}$ & $9.97 \times 10^{3}$ & 0.108 \\
\hline $4 \mathrm{~T}$ & 175 & 1.51 & $99.5 \times 10^{3}$ & $5.15 \times 10^{3}$ & 0.089 \\
\hline $9 \mathrm{~T}$ & 300 & 2.15 & $93.3 \times 10^{3}$ & $4.62 \times 10^{3}$ & 0.115 \\
\hline $14 \mathrm{~T}$ & 330 & 2.23 & $75.3 \times 10^{3}$ & $5.22 \times 10^{3}$ & 0.140 \\
\hline
\end{tabular}

reduction factor $R_{T \mathrm{e}}$ varies from 0.50 to 0.90 with an increment of 0.05 . The estimated parameters and flow dimension with the accuracy of three significant digits are all the same for different values of $R_{T \mathrm{e}}$, indicating that the parameter estimation is independent of $R_{T \mathrm{e}}$.

\subsection{Hydrogeologic interpretation}

One may expect that the drawdown response at a point adjacent to a pumping well might interpret the fracture flow system as a linear system. In contrast, for a point far away from the pumping well, the flow tends to be cylindrical. The 

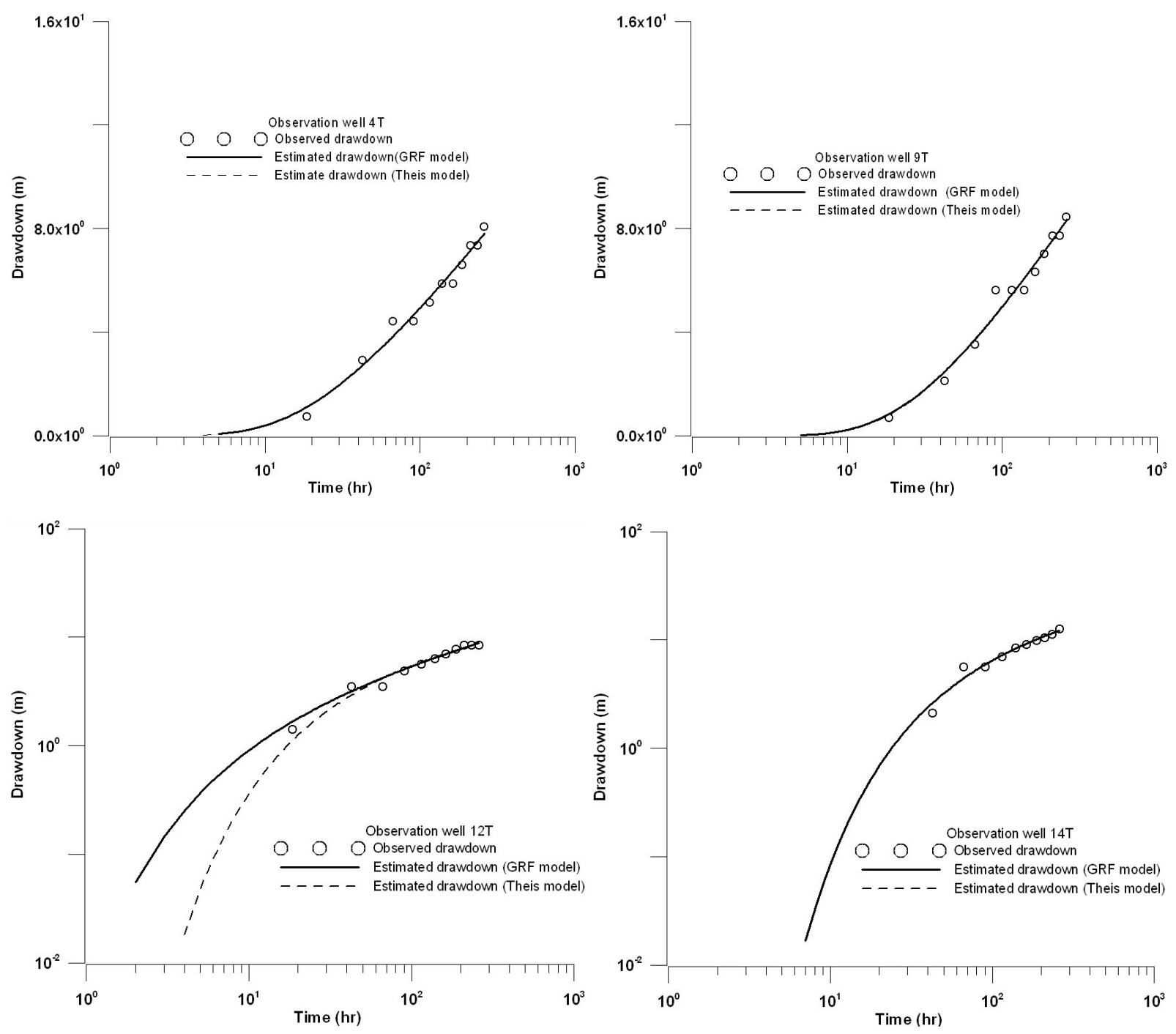

Fig. 5. The drawdowns for different models: Observation well (a) $4 \mathrm{~T}$; (b) $9 \mathrm{~T}$; (c) $12 \mathrm{~T}$; and (d) $14 \mathrm{~T}$.

analyzed results obtained from the proposed approach indicate that the flow dimension of the fracture zone between wells $12 \mathrm{~T}$ and $16 \mathrm{~T}$ is about 1.31 , implying that the fracture flow displays the characteristic of linear or elliptical flow in the region near well $12 \mathrm{~T}$. On the other hand, the estimated flow dimensions are 1.97, 2.11 and 2.27 for the data obtained from the wells $4 \mathrm{~T}, 9 \mathrm{~T}$ and $14 \mathrm{~T}$, respectively. The pressure response at well $4 \mathrm{~T}$ demonstrates the characteristic of radial flow, which is indeed the Theis' flow. The pressure responses at wells $9 \mathrm{~T}$ and $14 \mathrm{~T}$ show the flow varying from cylindrical toward spherical It clearly exhibits that the flow dimension of CGF increases with the distance between pumping well and observation well. Naturally, the increase with the flow distance also reflects the complexity of fracture orientation and interconnectivity of the rock mass and thus the variability of flow direction in a fractured medium as well. Le Borgne et al. (2004) investigated the time series of drawdowns which were recorded in piezometers located at distances ranging from 2 to $400 \mathrm{~m}$ from the pumping well and within the period ranging from 5 to 88 days. They analyzed short-, medium-, and long-term pumping test data sets using the GRF model to determine the flow dimensions. The short-term pumping test in seven wells lasted for 5 days, the medium-term test in two wells lasted for 13 days and the long-term test in two wells lasted for 88 days. Their results show that the estimated flow dimensions lie in the range from 1.4 to 1.7 and there is no obvious relation between the flow dimension and the distance from the pumping well. Their results and conclusions may however not be valid if they are based on the following two conditions:

\subsubsection{Geological features}

The site chosen to perform the data analysis in their study was located at the contact of two main tectonic features. One 

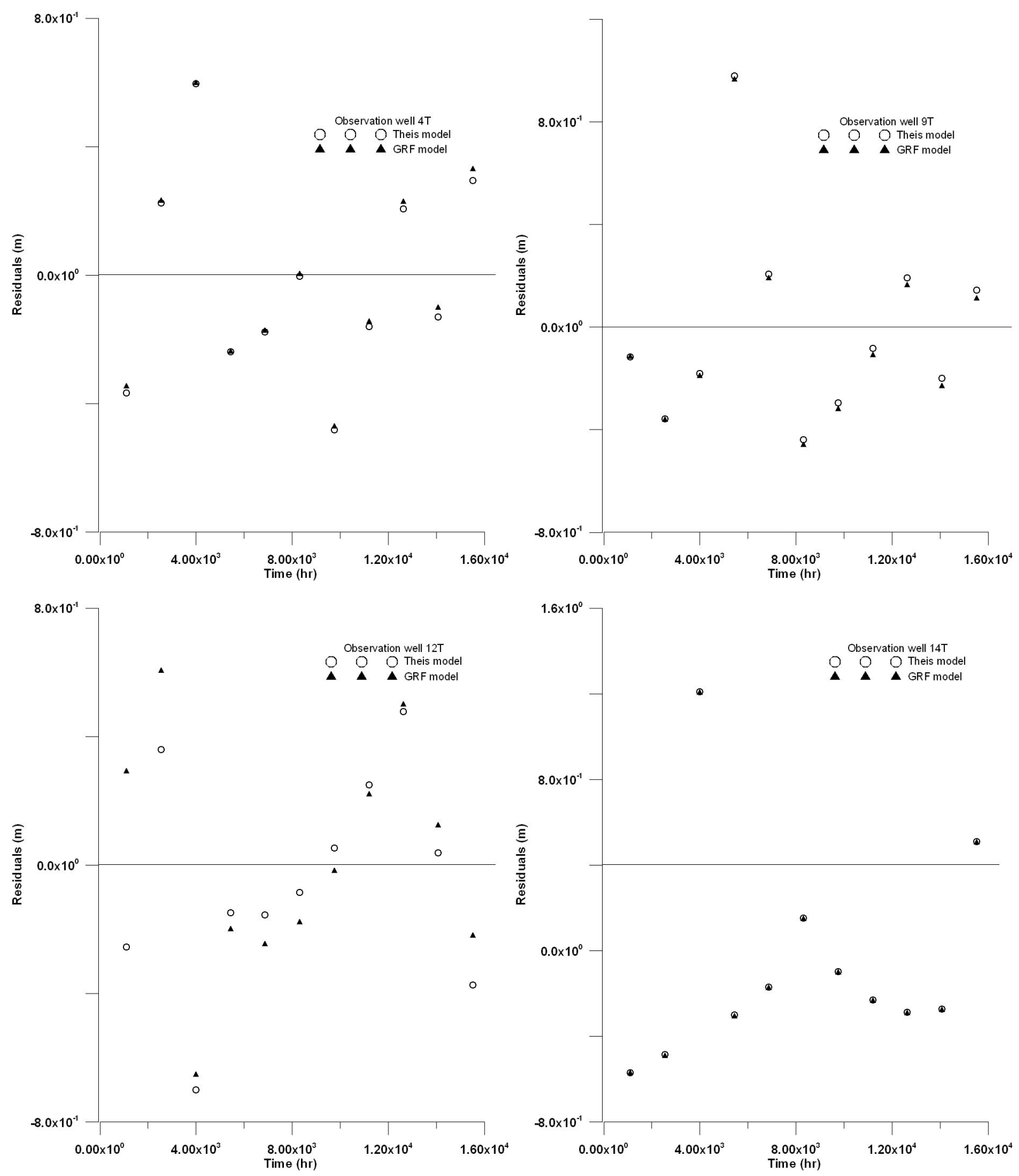

Fig. 6. The residuals for different models: Observation well (a) $4 \mathrm{~T}$; (b) $9 \mathrm{~T}$; (c) $12 \mathrm{~T}$; and (d) $14 \mathrm{~T}$.

was a regional contact between granite and schist while the other had two parallel faults that shift the contact zone. The contact zone was characterized by an alternation of schist enclaves and granitic dykes of aplites and pegmatites. All the pumping wells and piezometers were located at this region. It is not surprised that the estimated flow properties are characterized in the transition between linear and radial flows (i.e. the flow dimension ranging from 1.4 to 1.7 ) in their study since the flow regime may be strongly influenced by these two parallel faults. According to the tectonic descriptions mentioned above, this site is highly heterogeneous, which may seriously violate the homogeneous assumption of 


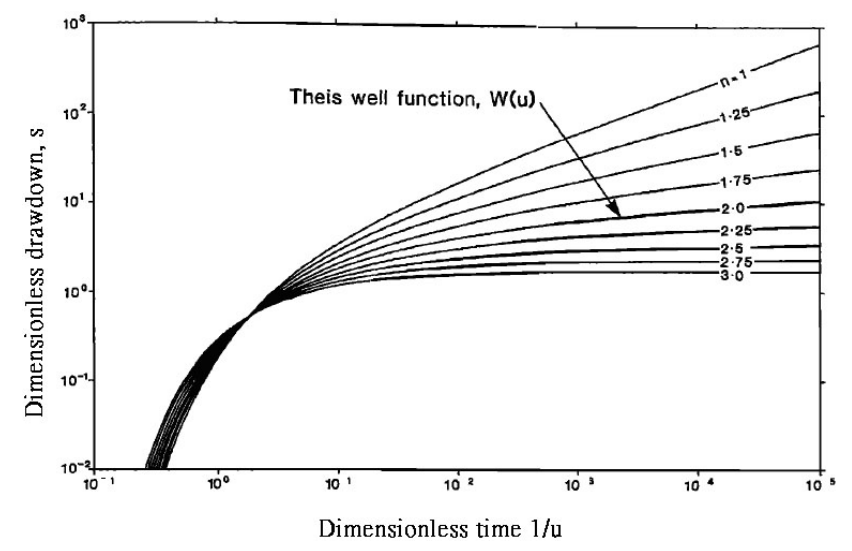

Fig. 7. Dimension drawdown and time curves (Modified from Fig. 2 in Barker (1988)).

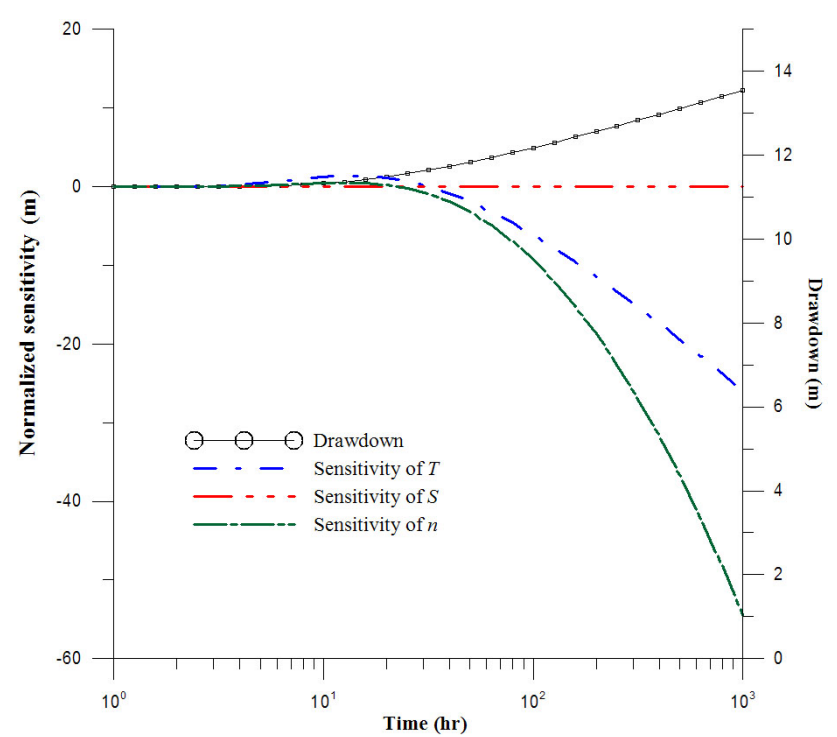

Fig. 8. The time-drawdown data and the sensitivities of the hydraulic parameters $T, S$ and the flow dimension $n$.

the GRF model. The validity of their estimated flow dimensions and hydrogeologic properties is thus questionable.

\subsubsection{Fitting models}

Le Borgne et al. (2004) used a graphical fitting procedure to determine the flow dimension for the data obtained from a long-term test. They fitted the asymptotic model for medium- and long-term data sets based on the infinite time assumption. The incomplete gamma function in Eq. (1) can be expressed as

$\Gamma(-v, u)=-\frac{1}{v} \Gamma(1-v)+\frac{u^{-1}}{v} M(-v, 1-v,-u)$

where $M(a, b, x)$ is the Kummer's function which has the value of 1 when $x$ tends to zero (Abramowitz and Stegun,
Table 5. The estimated values of hydrogeologic parameters for $4 \mathrm{~T}$, $9 \mathrm{~T}, 12 \mathrm{~T}$ and $14 \mathrm{~T}$ using various temperature reduction factor $R_{T \mathrm{e}}$ varies from 0.50 to 0.90 with an increment of 0.05 .

\begin{tabular}{cccc}
\hline \multicolumn{4}{c}{$4 \mathrm{~T}$} \\
\hline$R_{T \mathrm{e}}$ & $T\left(\mathrm{~m}^{2} \mathrm{~min}^{-1}\right)$ & $S$ & $n$ \\
$0.50 \sim 0.9$ & $48.9 \times 10^{-3}$ & $5.13 \times 10^{-3}$ & 1.95 \\
\hline \multicolumn{5}{c}{$9 \mathrm{~T}$} \\
\hline$R_{T \mathrm{e}}$ & $T\left(\mathrm{~m}^{2} \mathrm{~min}^{-1}\right)$ & $S$ & $n$ \\
$0.50 \sim 0.9$ & $71.2 \times 10^{-3}$ & $3.64 \times 10^{-3}$ & 2.11 \\
\hline \multicolumn{5}{c}{$12 \mathrm{~T}$} \\
\hline$R_{T \mathrm{e}}$ & $T\left(\mathrm{~m}^{2} \mathrm{~min}^{-1}\right)$ & $S$ & $n$ \\
$0.50 \sim 0.9$ & $99.9 \times 10^{-3}$ & $9.99 \times 10^{-3}$ & 1.31 \\
\hline$R_{T \mathrm{e}}$ & $T\left(\mathrm{~m}^{2} \mathrm{~min}^{-1}\right)$ & $S$ & $n$ \\
$0.50 \sim 0.9$ & $96.0 \times 10^{-3}$ & $6.54 \times 10^{-3}$ & 2.27 \\
\hline
\end{tabular}

1965). Thus, the asymptotic form of Eq. (1) is

$h(r, t)=\frac{Q_{0} r^{2 v}}{4 \pi^{1-\nu} \mathrm{Kb}^{3-n} v}\left[\left(\frac{4 \mathrm{Kt}}{\mathrm{Sr}^{2}}\right)^{v}-\Gamma(1-v)\right]$

The dimensionless form of Eq. (16) obtained using the definitions of dimensionless drawdown and dimensionless time in Le Borgne et al. (2004) is

$s^{*}=\frac{1}{v}\left[\left(t^{*}\right)^{v}-\Gamma(1-v)\right]$

where

$s^{*}=\frac{h(r, t)}{\left[\frac{Q_{0} r^{2 v}}{4 \pi^{1-\nu} \mathrm{Kb}^{3-n}}\right]}$ and $t^{*}=\left(\frac{4 \mathrm{Kt}}{\mathrm{Sr}^{2}}\right)$

Eq. (18) was simplified in Le Borgne et al. (2004) by neglecting the second right-hand-side term as:

$n=2 \times\left[1-\frac{\mathrm{d} \log s}{\mathrm{~d} \log t}\right]$

They fitted the medium- and long-term data using Eq. (19). As shown in Fig. 9, there is a large difference between the asymptotic and exact dimensionless drawdown, especially in the cases of small dimensionless time and/or large $n$. In Le Borgne et al. (2004), the range of flow dimension is from 1.4 to 1.7 and there are distinct differences between asymptotic and exact dimensionless drawdown in this range of flow dimension. The accuracies of their estimated flow dimension and hydrogeologic parameters are therefore questionable. 


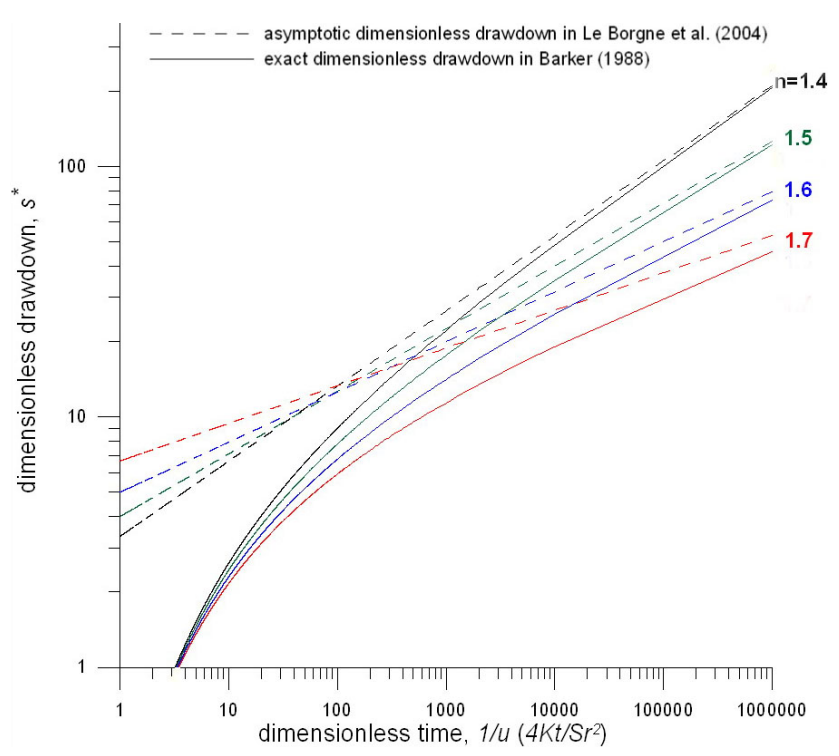

Fig. 9. Dimensionless drawdown versus dimensionless time for flow dimension $n$ varying from 1.4 to 1.7 .

\section{Concluding remarks}

This study first develops an approach, combined the GRF model with a heuristic optimization scheme, SA, for determining the fractional flow dimension and hydrogeologic parameters of the fractured medium. The measured drawdowns obtained from four observation wells during an 11-day long hydraulic test performed at CGF in Taiwan are then chosen for the data analysis using the present approach. The results demonstrate that the present approach can successfully determine the flow dimension and hydrogeologic parameters of the CGF fractured formation. We found that the flow dimension increases with the distance between the pumping well and the observation well. This paper provides a useful approach and a case study in analyzing field pumping test data obtained from fractured formations for simultaneously determining the flow dimension and hydrogeologic parameters. We hope that this paper can stimulate further research on the topic of scale-dependent effect on flow dimension of fractured media.

Acknowledgements. Research leading to this work has been partially supported by the grants from Taiwan National Science Council under the contract numbers NSC99-2221-E-009-062MY3, NSC 99-NU-E-009-001 and the "Aim for the Top University Plan" of the National Chiao Tung University and Ministry of Education, Taiwan.

Edited by: A. Guadagnini

\section{References}

Abramowitz, M. and Stegun, I. A.: Handbook of Mathematical Functions, Dover publications, New York, 1970.

Acuna, J. A. and Yortsos, Y. C.: Application of fractal geometry to the study of networks of fractures and their pressure transient, Water Resour. Rese., 31, 527-540, doi:10.1029/94WR02260, 1995.

Audouin, O. and Bodin, J.: Cross-borehole slug test analysis in a fractured limestone aquifer, J. Hydrol., 348, 510-523, doi:10.1016/j.jhydrol.2007.10.021, 2008.

Barker, J. A.: A generalized radial flow model for hydraulic tests in fractured rock, Water Resour. Res., 24, 1796-1804, doi:10.1029/WR024i010p01796, 1988.

Berthouex, P. M. and Brown, L. C.: Statistics for Environmental Engineers, 2nd Edition, Lewis Publishers, USA, 2002.

Butler Jr, J. J. and Zhan, X.: Hydraulic tests in highly permeable aquifers, Water Resour. Res., 40, W12402, doi:10.1029/2003WR002998, 2004.

Chang, C. R. Y. and Ramey, H. J.: Well interference test in the Chingshui geothermal field, In 5th Geothermal Reservoir Engineering Workshop, Stanford University, Stanford, Calif., 1979.

Chang, J. and Yortsos, Y. C.: Pressure-transient analysis of fractal reservoirs, SPE Form Eval., 5(631), 31-38, doi:10.2118/18170PA, 1990.

Chen, C. S. and Liu, I. Y.: Comment on "Flow dimensions corresponding to hydrogeologic conditions" by Douglas D. Walker and Randall M. Roberts, Water Resour. Res., 43, W02601, doi:10.1029/2006WR005435, 2007.

Chakrabarty, C.: A note on fractional dimension analysis of constant rate interference tests, Water Resour. Res., 30, 2339-2341, doi:10.1029/94WR00759, 1994.

Chiang, S. C., Lin, J. J., Chang, C. R. Y., and Wu, T. M.: A preliminary study of the Chingshui geothermal area, Ilan, Taiwan, Paper presented at Fifth Geothermal Reservoir Engineering Workshop, Stanford University, Stanford, CA, 269-274, 1979.

Czarnecki, J. B., and Craig, R. W.: A program to calculate aquifer transmissivity from specific capacity data for programmable calculators, Ground Water, 23, 667-672, doi:10.1111/j.17456584.1985.tb01515.x, 1985.

Fan, K. F., Kuo, M. C. T., Liang, K. F., Lee, C. S., and Chiang, S. C.: Interpretation of a well interference test at the Chingshui geothermal field, Taiwan, Geothermics, 34, 99-118, doi:10.1016/j.geothermics.2004.11.003, 2005.

Hamm, S. Y. and Bidaux, P.: Dual-porosity fractal models for transient flow analysis in fissured rocks, Water Resour. Res., 32, 2733-2745, doi:10.1029/96WR01464, 1996.

Hsiao, P. T. and Chiang, S. C.: Geology and geothermal system of the Chingshui-Tuchang geothermal area, Ilan, Taiwan, Petrol, Geol, Taiwan, 16, 205-213, 1979.

Huang, Y. C. and Yeh, H. D.: The use of sensitivity analysis in on-line aquifer parameter estimation, J. Hydrol., 335, 406-418, doi:10.1016/j.jhydrol.2006.12.007,, 2007.

Huang, Y. C., Yeh, H. D., and Lin, Y. C.: A computer method based on simulated annealing to identify aquifer parameters using pumping test data, Int. J. Numer. Anal. Met., 32, 235-249, doi:10.1002/nag.623, 2008.

Jenkins, D. N. and Prentice, J. K.: Theory for aquifer test analysis in fractured rocks under linear (nonradial) flow conditions, Ground Water, 20, 12-21, doi:10.1016/j.advwatres.2009.03.009, 1982. 
Kirkpatrick, S., Gelatt, C. D. Jr, and Vecchi, M. P.: Optimization by simulated annealing, Science, 220, 671-680, 1983.

Kuusela-Lahtinen, A., Niemi, A., and Luukkonen, A.: Flow dimension as an indicator of hydraulic behavior in site characterization of fractured rock, Ground Water, 41, 333-341, doi:10.1126/science.220.4598.671, 2003.

Le Borgne, T., De Dreuzy, J. R., Davy, P., and Touchard, F.: Equivalent mean flow models for fractured aquifers: Insights from a pumping tests scaling interpretation, Water Resour. Res., 40, W03512, doi:10.1029/2003WR002436, 2004.

Leveinen, J.: Composite model with fractional flow dimensions for well test analysis in fractured rocks, J. Hydrol., 234, 116-141, 2000.

Leveinen, J., Ronka, E., Tikkanen, J., and Karro, E.: Fractional flow dimensions and hydraulic properties of a fracturezone aquifer, Leppavirta, Finland, Hydrogeol. J., 6, 327-340, doi:10.1007/s100400050156, 1998.

Lods, G. and Gouze, P.: A generalized solution for transient radial flow in hierarchical multifractal fractured aquifers, Water Resour. Res., 44, W12405, doi:10.1029/2008wr007125, 2008.

Metropolis, N., Rosenbluth, A. W., Rosenbluth, M. N., Teller, A. H., and Teller, E.: Equation of state calculations by fast computing machines, J. Chem. Phys., 21, 1087, doi:10.1063/1.1699114, 1953.

Mukhopadhyay, A.: Automated derivation of parameters in a nonleaky confined aquifer with transient flow, Ground Water, 23, 806-811, doi:10.1111/j.1745-6584.1985.tb01963.x, 1985.

Papadopulous, I. S.: Nonsteady flow to a well in an infinite anisotropic aquifer, Proceeding of the Durbrovnik Symposium on the Hydrology of Fractured Rocks, International Association of Scientific Hydrology, 21-31, 1965.

Pickens, J., Grisak, G., Avis, J., Belanger, D., and Thury, M.: Analysis and interpretation of borehole hydraulic tests in deep boreholes: principles, model development and application, Water Resour. Res., 23, 1341-1375, 1987.

Pham, D. T. and Karaboga, D.: Intelligent optimization techniques: genetic algorithms, tabu search, simulated annealing and neural networks, Springer-Verlag, New York, 2000.

Rafini, S. and Larocque, M.: Insights from numerical modeling on the hydrodynamics of non-radial flow in faulted media, Adv. Water Resour., 32, 1170-1179, doi:10.1016/j.advwatres.2009.03.009, 2009.

Rai, S. P.: Numerical determination of aquifer constants, J. Hydraul. Eng.-ASCE, 111, 1110-1114, doi:10.1061/(ASCE)07339429(1985)111:7(1110), 1985.
Rehbinder, G.: Analytical solutions for groundwater flow with arbitrary dimensionality and a finite well radius in fractured rock, Water Resour. Res., 46, W03531, doi:10.1029/2009WR008115, 2010.

Sen, Z.: Aquifer test analysis in fractured rocks with linear flow pattern, Ground Water, 24, 72-78, doi:10.1111/j.17456584.1986.tb01461.x, 1986.

Su, F. C.: Resistivity survey in the Chingshui prospect, I-Lan, Taiwan, Petroleum Geology of Taiwan, 15, 255-264, 1978.

Theis, C. V.: The relation between the lowering of the piezometric surface and the rate and duration of discharge of a well using ground-water storage, Trans., American Geophysical Union, 16, 519-524, 1935.

Tong, L.T., Ouyang, S., Guo, T. R., Lee, C. R., Hu, K. H.,Lee, C. L., and Wang, C. J.: Insight into the geothermal structure in Chingshui, Ilan, Taiwan, Terr. Atmos. Ocean. Sci., 19(4), 413424, doi:10.3319/TAO.2008.19.4.413(T), 2008.

Tseng, C. S.: Geology and geothermal occurrence of the Chingshui and Tuchang districts, Ilan. Petrol, Geol. Taiwan, 15, 11-23, 1978.

Verbovšek, T.: Influences of aquifer properties on flow dimensions in dolomites, Ground Water, 47, 660-668, doi:10.1111/j.17456584.2009.00577.x, 2009.

Walker, D. D. and Roberts, R. M.: Flow dimensions corresponding to hydrogeologic conditions, Water Resour. Res., 39, 1349, doi:10.1029/2002WR001511, 2003.

Walker, D. D., Cello, P. A., Valocchi, A. J, and Loftis, B.: Flow dimensions corresponding to stochastic models of heterogeneous transmissivity, Geophys. Res. Lett., 33, L07407, doi:10.1029/2006GL025695, 2006.

$\mathrm{Xu}, \mathrm{M}$. and Eckstein, Y.: Use of weighted least-Squares method in evaluation of the relationship between dispersivity and field scale, Ground Water, 33, 905-908, doi:10.1111/j.17456584.1995.tb00035.x, 1995.

Yeh, H. D.: Theis' solution by nonlinear least-squares and finitedifference Newton's method, Ground Water, 25, 710-715, doi:10.1111/j.1745-6584.1987.tb02212.x, 1987.

Yeh, H. D., Lin, Y. C., and Huang, Y. C.: Parameter identification for leaky aquifers using global optimization methods, Hydrol. Process., 21, 862-872, doi:10.1002/hyp.6274, 2007. 